We appreciate the response to this publication feature and welcome all contributions. Contributions may be sent to our Technical Editors: José A Mascorro,jmascor@hotmail.com or Phil Oshel, oshel1pe@cmich.edu

\section{Rhodamine Fluorescence After 15-year Storage in Methyl Salicylate}

\author{
Philip L. Hertzler \\ Dept. of Biology, Central Michigan University \\ hertz1pl@cmich.edu
}

Fading of fluorochrome is a significant limitation to fluorescence microscopy. Several anti-fade agents, e.g. n-propyl gallate, are commonly used for glycerol-based mounting media (Longin et al., 1993; Ono et al., 2001). Samples mounted in glycerol must be kept at $-20^{\circ} \mathrm{C}$ for long-term storage to prevent bacterial degradation. In contrast, fluorescent samples cleared and mounted in organic media can be stored indefinitely at room temperature.

Methyl salicylate or oil of wintergreen is an excellent clearing agent (refractive index $=1.53$ ), which works well with a variety of fluorochromes. It has a pleasant aroma but is somewhat difficult to work with since it remains liquid after mounting. It was previously reported that shrimp embryos labeled with tubulin antibody and rhodamine-conjugated secondary antibody maintained their fluorescence after six months (Summers et al. 1993). These same samples, stained in November, 1990 and imaged by confocal microscopy for publication in Hertzler and Clark (1992), are still fluorescent after continuous storage in methyl salicylate at room temperature in the dark (Figure 1). The images of 62-cell stage shrimp embryos taken from these 1990 samples were collected with an Olympus Fluoview 300 laser scanning confocal microscope in January, 2006 in the Dept. of Biology, Central Michigan University. There appears to be no loss of resolution in the high magnification image (Figure 1B). Samples I have stained with $\mathrm{Cy} 2$, Cy3, BODIPY FL, Alexa Fluor 546, and Sytox Green and mounted in methyl salicylate have also retained fluorescence for months or years. Therefore, in addition to its excellent clearing properties, methyl salicylate is also useful for maintaining fluorescence during long-term storage of thick specimens for confocal microscopy.
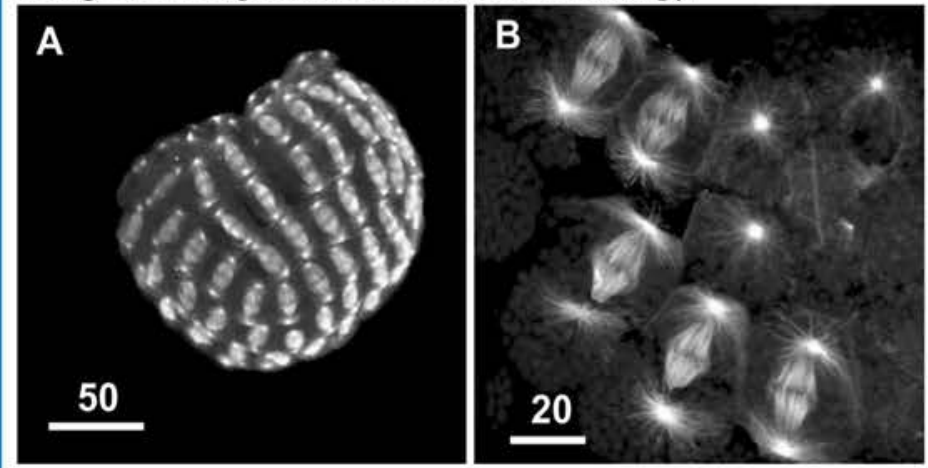

Figure 1 Optical sections of 62-cell stage shrimp embryo whole mounts labeled with E7 anti- $\beta$-tubulin antibody and rhodomine-conjugated secondary antibody. (A) Extended focus of sections 1-15 of 30 through entire embryo, step size $3 \mu \mathrm{m}$, using Olympus $20 \mathrm{X} 0.7 \mathrm{NA}$ Plan-Apochromat objective, zoom $=3$. (B) Extended focus of sections 3-5 of 10 through single cells, step size $1 \mu \mathrm{m}$, using Olympus 60X 1.4 NA Plan-Apochromat objective, zoom $=2$. Scale bars in micrometers.

\section{Acknowledgements:}

I thank Phil Oshel, Dept. of Biology, Central Michigan University, for encouraging me to submit this observation for publication. The E7 anti- $\beta$-tubulin antibody developed by $\mathrm{M}$. Klymkowsky was obtained from the Developmental Studies Hybridoma Bank maintained by The University of Iowa, Department of Biological Sciences, Iowa City, IA 52242.

\section{References:}

1. Hertzler, P.L. and Clark, W.H., Jr. Cleavage and gastrulation in the shrimp Sicyonia ingentis: invagination is accompanied by oriented cell division, (1992) Development 116, 127-140.

2. Longin A., Souchier, C., Ffrench, M. and Bryon, P.A. Comparison of anti-fading agents used in fluorescence microscopy: image analysis and laser confocal microscopy study, (1993). J. Histochem. Cytochem. 41, 1833-1840.

3. Ono, M., Murakami, T., Kudo, A., Isshiki, M., Sawada, H., and Segawa, A. Quantitative comparison of anti-fading mounting media for confocal laser scanning microscopy, (2001) J. Histochem. Cytochem. 49, 305-312.

4. Summers, R.G., Sticker, S.A. and Cameron, R.A. Applications of confocal microscopy to studies of sea urchin embryogenesis, (1993) In: Matsumoto, B. (ed.) Methods in Cell Biology 38, 265-287.

\section{When Point To Point Is Not Enough}

\section{Carol Heckman, Marilyn Cayer, Mita Varghese \\ Center for Microscopy \& Microanalysis, Bowling Green State University, Bowling Green, $\mathrm{OH}$ heckman@bgnet.bgsu.edu}

As all microscopists are taught, resolution can be measured by knowing the closest distance between two points that can be discriminated in the image. There are some occasions when we want to measure something in a digital image, and the nominal resolution isn't sufficient for the task. One of these, extracting a contour from the image, was summarized in a previous research report [1]. The principles would be valid for any filled area, such as a particle or structure from an X-ray dot map. The ground rule was set that the outermost pixel would be selected at every point on the contour. It was clear from the geometry shown in Fig. 1 that only values of $0^{\circ}, 45^{\circ}, 90^{\circ}, 135^{\circ}$ and $180^{\circ}$ could be measured. Because of the stair step defect, tracing the sequence of pixel locations only gave offsets to 8 pixels, one directly below, two on the corners below, three pixels in the same location above, and one pixel on either side of the subject pixel. Therefore it was impossible to measure the true curvature of the contour.
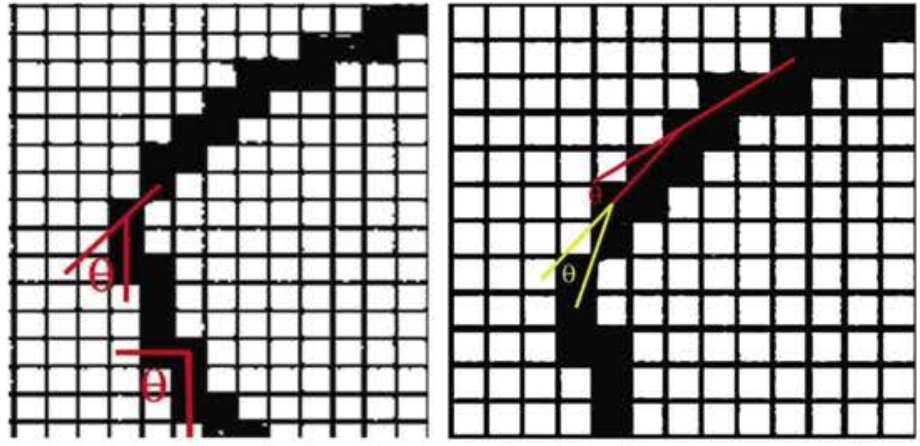

Fig. $1.45^{\circ}$ and $90^{\circ}$ angles generated without dropping points on the contour

Fig. 2. $13^{\circ}$ (red) and $19^{\circ}$ (yellow) angles generated after point dropping.

The solution to these difficulties was point dropping. The ideal number of points that must be dropped in order to get an accurate curvature measurement was determined [2]. The rule that two pixels be dropped for every pixel accepted gave a curvature measurement that was continuously variable between $0^{\circ}$ and $180^{\circ}$ (Fig. 2). Moreover the curvature calculated for an actual test figure differed from the theoretical by only $1 \%$. The solution has been used frequently to solve biological 